\title{
Outcome measurement instruments for peripheral vascular malformations and an assessment of the measurement properties: a systematic review
}

\author{
Sophie E. R. Horbach ${ }^{1} \cdot$ Amber P. M. Rongen ${ }^{1} \cdot$ Roy G. Elbers ${ }^{2}$. Chantal M. A. M. van der Horst ${ }^{1}$. Cecilia A. C. Prinsen ${ }^{3}$. \\ Phyllis I. Spuls ${ }^{4}$. Collaborators OVAMA consensus group
}

Accepted: 9 September 2019 / Published online: 23 September 2019

(c) The Author(s) 2019

\begin{abstract}
Purpose The Outcome measures for vascular malformation (OVAMA) group reached consensus on the core outcome domains for the core outcome set (COS) for peripheral vascular malformations (venous, lymphatic and arteriovenous malformations). However, it is unclear which instruments should be used to measure these domains. Therefore, our aims were to identify all outcome measurement instruments available for vascular malformations, and to evaluate their measurement properties.

Methods With the first literature search, we identified outcomes and instruments previously used in prospective studies on vascular malformations. A second search yielded studies on measurement properties of patient- and physician-reported instruments that were either developed for vascular malformations, or used in prospective studies. If the latter instruments were not specifically validated for vascular malformations, we performed a third search for studies on measurement properties in clinically similar diseases (vascular or lymphatic diseases and benign tumors). We assessed the methodological quality of these studies following the Consensus-based Standards for the selection of health Measurement Instruments methodology, and evaluated the quality of the measurement properties.

Results The first search yielded 27 studies, none using disease-specific instruments. The second and third search included 22 development and/or validation studies, concerning six instruments. Only the Lymphatic Malformation Function Instrument was developed specifically for vascular malformations. Other instruments were generic QoL instruments developed and/or partly validated for clinically similar diseases.

Conclusions Additional research on measurement properties is needed to assess which instruments may be included in the COS. This review informs the instrument selection and/or the development of new instruments.
\end{abstract}

Systematic review registration PROSPERO, 42017056242.

Keywords Vascular malformations · Outcome instruments - Outcome measurement instruments · Patient reported · PROMs $\cdot$ Physician reported $\cdot$ Measurement properties $\cdot$ Validity $\cdot$ Reliability $\cdot$ Responsiveness $\cdot$ Interpretability

Sophie E. R. Horbach and Amber P. M. Rongen contributed equally to this work.

The Collaborators OVAMA consensus group are listed in "Acknowledgements".

Electronic supplementary material The online version of this article (https://doi.org/10.1007/s11136-019-02301-x) contains supplementary material, which is available to authorized users.

Sophie E. R. Horbach

s.e.horbach@amsterdamumc.nl

Extended author information available on the last page of the article

\section{Introduction}

Vascular malformations are benign congenital anomalies of the vascular system that are categorized based on the type of vascular channels involved: arterial, venous, lymphatic or capillary vessels, singularly or combined [1-4]. The disease impact varies, including cosmetic concerns, pain and functional impairment, depending on the size and location of the lesion. Multiple treatment options are available, including compressive stockings, sclerotherapy and surgery; however, evidence is lacking to support clinical decision-making. 
In current literature, there is no uniformity regarding the outcomes that are measured to evaluate treatment effectiveness ('outcome domains') and what measurement methods or tools are used to measure these outcomes (also known as 'outcome measurement instruments' or simply 'instruments'. For this reason, outcomes of different clinical trials cannot be pooled, which hampers the development of evidencebased guidelines.

The Outcome Measures for VAscular MAlformations (OVAMA) group initiated the OVAMA project aiming to develop an international 'Core Outcome Set' (COS) for adult as well as pediatric patients with peripheral congenital soft tissue vascular malformations for measuring outcomes of therapeutic interventions globally. A COS is an agreed minimum set of outcomes that should be measured and reported in all clinical trials of a specific disease or trial population [5]. In an earlier e-Delphi study, the OVAMA consensus group reached consensus on the core outcome domains for patients with venous, lymphatic, and arteriovenous malformations (VM, LM, and AVM respectively): radiological assessment, physician-assessed location-specific signs, patient-reported pain, overall severity of symptoms, health-related quality of life (HRQoL), patient satisfaction with treatment and outcome, and adverse events (Online Resource 1). For each unique type of vascular malformation, specific physician- and patient-reported signs and symptoms were included separately. Furthermore, recurrence and appearance were recommended outcome domains based on the e-Delphi study but require further discussion before final inclusion in the COS [6]. However, it is unclear which instruments are most suitable for measuring the core outcome domains in adults and children.

This review, as part of the OVAMA project (Online Resource 2), aims to identify the outcome domains and instruments for vascular malformations that were used in previous prospective studies, and to assess the quality of the available patient- and physician-reported outcome instruments, to inform the selection process of instruments to measure the core outcome domains in future studies.

\section{Materials and methods}

The OVAMA project was registered at the Core Outcome in Effectiveness Trials (COMET) database (http://www.comet -initiative.org/), designed following the Harmonizing Outcome Measures for Eczema (HOME) roadmap, and embedded within the Cochrane Skin Group-Core Outcome Set Initiative (CSG-COUSIN) [7]. We followed the guidelines of the PRISMA-P statement [8], the Core Outcome SetSTAndards for Reporting (COS-STAR) [9], and the COnsensus-based Standards for the selection of health Measurement INstruments (COSMIN) 'Protocol for Systematic Reviews of Measurement Properties' [10]. The study protocol was registered in PROSPERO (42017056242).

\section{Literature searches, study selection and data extraction}

This systematic review consisted of three literature searches (Online Resource 3), as described below.

All searches were performed with the help of a clinical librarian. The PubMed function 'Similar Articles' and reference lists of all included articles were screened for additional studies. Study selection and data extraction were performed by two independent reviewers. Disagreements were resolved by consensus.

\section{Search 1: identification and description of outcome domains and instruments used in previously published studies}

In the first search, prospective studies evaluating treatment outcomes in vascular malformations were identified to collect all outcome domains and instruments that were previously used. We searched MEDLINE, EMBASE and CENTRAL for studies measuring treatment effectiveness, using search terms for peripheral vascular malformations. We included prospective studies evaluating treatments that enrolled at least 20 patients (all ages) with all singular and combined types of vascular malformations. Capillary, visceral, bone and central nervous system vascular malformations were excluded as these were outside the scope of the OVAMA project. Articles published before 1996 were excluded, since the current classification and terminology for vascular anomalies was established in 1996 by the International Society for the Study of Vascular Anomalies (ISSVA) [11]. Data on study characteristics, outcome domains and instruments were extracted.

\section{Searches 2 and 3: evaluation of the quality of the identified outcome measurement instruments}

The second search was performed in MEDLINE and EMBASE to identify development and validation studies of patient- and physician-reported disease-specific instruments for vascular malformations, using search terms for vascular malformations and a validated PubMed search filter for finding studies on measurement properties, developed by Terwee et al. [12].

The third search aimed to identify development and validation studies of patient- and physician-reported outcome measurement instruments that were used in previously published prospective studies on vascular malformations, as identified in search 1 , but were not specifically developed and validated for vascular malformations. To explore 
their potential applicability in vascular malformations, we additionally searched for studies on measurement properties in patient populations that share clinical similarities with vascular malformations, predefined as: 'benign vascular diseases', 'benign lymphatic diseases' and 'benign soft tissue tumors'. The expert group considered these groups of health conditions to have the greatest similarity to vascular malformations in terms of clinical appearance, signs, symptoms and potential disease burden. Search terms for the identified patient- and physician-reported instruments were combined with terms for the predefined clinically similar diseases, and the abovementioned PubMed filter for studies on measurement properties [12]. Only studies reporting on at least one measurement property of an instrument that was developed for vascular malformations, or for a clinically similar condition but previously used for vascular malformations, were included. Studies not reporting on measurement properties and studies focusing on health conditions other than vascular malformations or the predefined clinically similar conditions were excluded.

Data on characteristics of the included instruments, study samples and the study results concerning measurement properties were extracted.

\section{Evaluation of the methodological quality of the included studies}

The methodological quality of the included studies on measurement properties was evaluated by two authors independently using the COSMIN checklist (www.cosmi n.nl) $[13,14]$. With this checklist, we evaluated, for each included study separately, which measurement properties were investigated (following the COSMIN taxonomy, Online Resource 4) and if the methods to do so were appropriate. Several items per measurement property were rated using on a 4-point rating scale ranging from 'excellent' to 'poor'. The overall score for each measurement property was determined by the 'worst score counts' principle [15]. As a gold standard for patient-reported outcome measures is lacking, criterion validity was not considered. Data on interpretability and feasibility were collected if presented in the included studies.
Two reviewers independently extracted data on the measurement properties from the selected articles and evaluated methodological quality of the studies. Discrepancies were discussed with a third reviewer until consensus was reached.

\section{Evaluation of quality of the measurement properties}

Two authors independently evaluated the quality of the measurement properties by rating the results of the analyses on measurement properties in each included study based on the criteria for good measurement properties as recommended by Terwee et al. [16, 17] (Online Resource 5). The study results were independently rated by two reviewers as 'positive' (+), 'negative' (-) or 'indeterminate' (?) for each measurement property.

\section{Best evidence synthesis}

The best evidence synthesis is aimed at reaching a conclusion about the overall quality of each of the measurement properties of the included instruments. For this purpose, the quality assessments of the measurement properties based on the study results of the included studies were combined and adjusted for the methodological quality of the studies by applying levels of evidence as recommended by the COSMIN group [16], taking into account the number of studies, the methodological quality of the studies and the consistency of the study results on measurement properties across studies.

For each measurement property, the methodological quality of the study (poor, fair, good or excellent) and the direction of the study results of the analyses on this measurement property (negative, indeterminate or positive result) were combined into the best evidence synthesis (Table 1):,,++++++ : positive rating indicating "adequate" measurement property; ?: unknown rating indicating indeterminate measurement property; - - -, - - , - : negative rating indicating "inadequate" measurement property; \pm : conflicting findings; NI: not interpretable

Table 1 Levels of evidence for the overall quality of a measurement property (www.cosmin.nl) [47]

\begin{tabular}{lll}
\hline Level & Rating $^{\mathrm{a}}$ & Criteria \\
\hline Strong & +++ or --- & Consistent findings in multiple studies of at least good quality OR one study of excellent quality \\
Moderate & ++ or - & $\begin{array}{c}\text { Consistent findings in multiple studies of fair methodological quality OR one study of good } \\
\text { methodological quality }\end{array}$ \\
Limited & + or - & One study of fair methodological quality \\
Conflicting & \pm & Conflicting findings \\
Unknown & $?$ & Only studies of poor methodological quality \\
\hline
\end{tabular}

${ }^{\mathrm{a}}+$ positive/good, ? indeterminate, - negative/poor, \pm conflicting ratings 
(due to indeterminate result of analysis); NA: not available. (analysis was not performed for this measurement property).

\section{Results}

\section{Search 1: identification of outcome domains and instruments}

In 26 of the 27 studies identified by search 1 (Fig. 1) the authors exclusively used outcomes that were recommended or selected as core outcome domains in the OVAMA e-Delphi study [6], however, inconsistently across the studies, measuring at least one of the following: adverse events [100\% of studies], radiological assessment [56\%], appearance [52\%], patient-reported symptoms including pain [37\%], patient satisfaction with treatment and/or outcome [26\%], physician-reported signs [15\%], HRQoL [15\%] and recurrence [7\%]. In one study, 'healthcare costs' was the primary outcome, categorized under the domain practical issues [4\%] [18], which was not selected as a core domain in the OVAMA e-Delphy study. All instruments used for each outcome domain are listed in Table 2. None of these were diseasespecific instruments for vascular malformations. Published 'named' instruments were only available for the assessment of HRQoL. Instruments for other outcome domains were unnamed questionnaires that were only created for singular use by the authors of the concerning study.

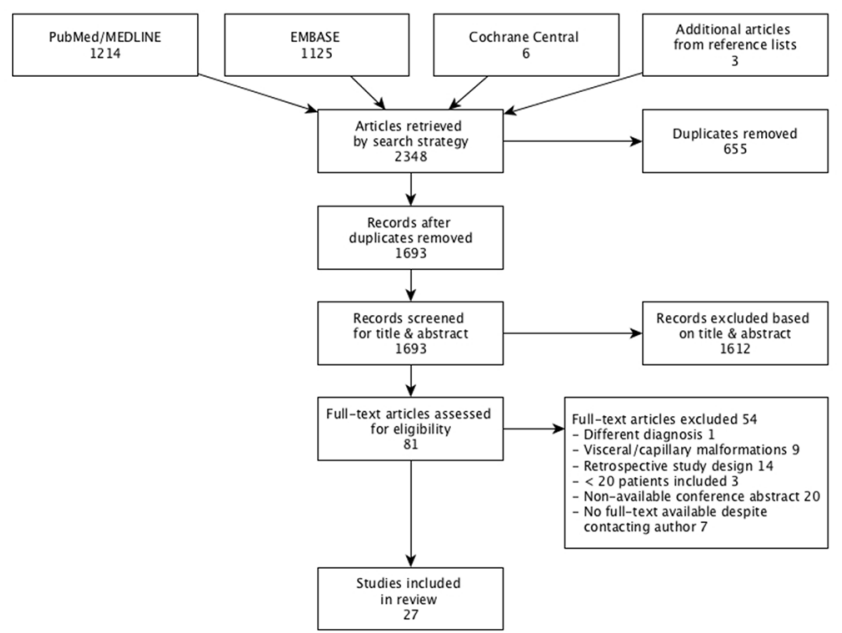

Fig. 1 Flowcharts of study selection. Search 1 (left): the identification of instruments previously used in prospective studies on vascular malformations. Search 2 and 3 (right): the identification of development and/or validation studies of disease-specific instruments for vas-

\section{Searches 2 and 3: evaluation of the quality of the identified instruments}

The searches for development and validation studies on instruments used for vascular malformations provided 4446 articles (vascular malformations $n=3170$; similar diseases $n=1276$ ), the major reasons for exclusion were failure to report on measurement properties and studies investigating unrelated health conditions. Twenty-two studies were included [19-40] evaluating six different instruments (Fig. 1), of which there was only one disease-specific instrument for vascular malformations. The other included instruments were four generic instruments developed or partly validated for other clinically similar diseases (two including a disease-specific module) and one disease-specific instrument for varicose veins, all previously used in vascular malformation studies. Characteristics of the selected instruments and study populations are presented in Tables 3 and 4 , respectively. The methodological quality of the studies, the quality of the measurement properties and the best evidence synthesis are presented in Table 5. Details on the COSMIN ratings and the evaluation of the measurement properties per included study can be found in Online Resource 6 .

\section{Lymphatic malformation function instrument (LMF)}

The LMF is a disease-specific questionnaire to assess functional and clinical signs and subsequent impact on daily life in pediatric patients with cervicofacial LMs. We found strong evidence for adequate content validity [40]. Because it was not described how missing values were handled, there was limited evidence for adequate internal consistency, for

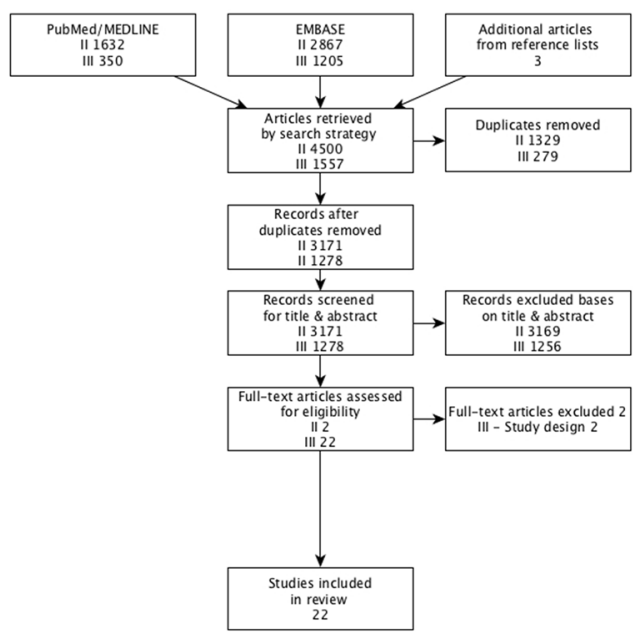

cular malformations (II), and development and/or validation studies for instruments previously used in prospective studies on vascular malformations (III) 


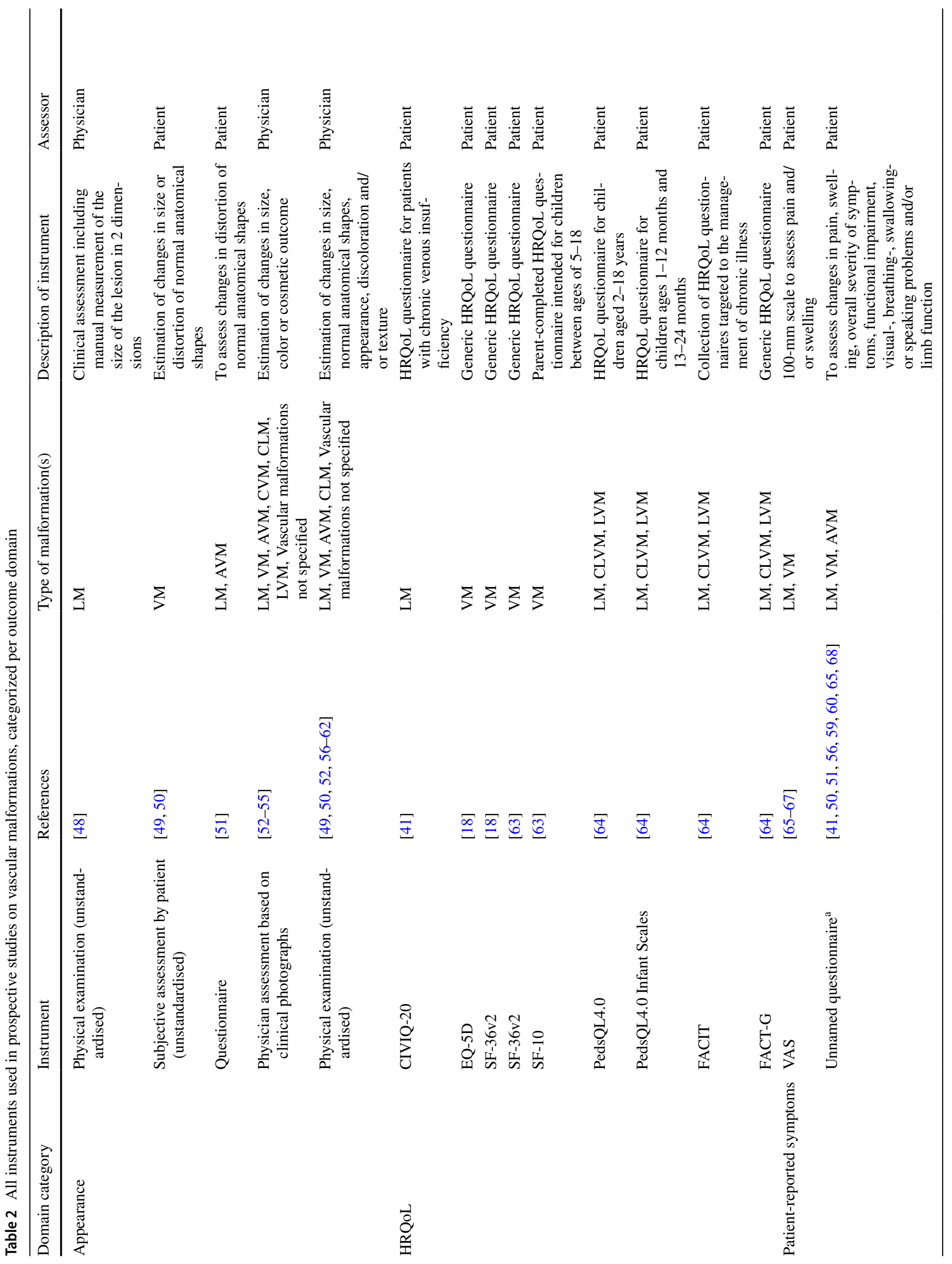




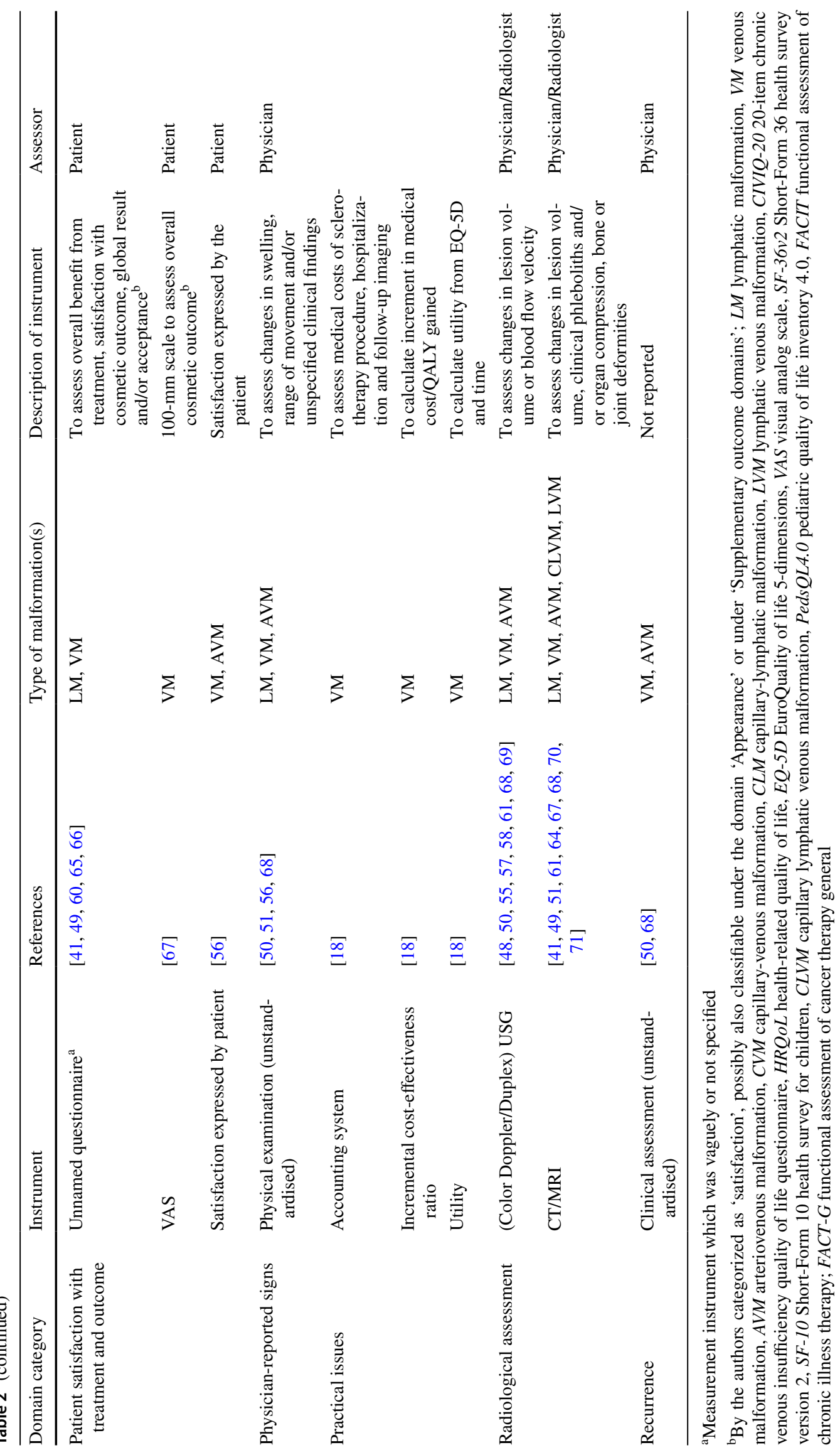


Table 3 Characteristics of the identified 'named' patient- and physician-reported outcome measurement instruments that were developed or previously used for vascular malformations, for which studies on measurement properties were available

\begin{tabular}{|c|c|c|c|c|c|c|}
\hline Instrument & Target population & Domains & No. of items & Scoring method & Total score range & $\begin{array}{l}\text { Recall } \\
\text { period } \\
\text { (days) }\end{array}$ \\
\hline $\operatorname{LMF}[27,40]$ & $\begin{array}{l}\text { Cervicofacial lym- } \\
\text { phatic malformations }\end{array}$ & $\begin{array}{l}\text { Functional impairment } \\
\text { (signs and impacts) }\end{array}$ & 12 & $\begin{array}{l}\text { 3-point Likert scale, } \\
\text { ordinal }\end{array}$ & $\begin{array}{l}0-2 \text {, with } 0=\text { best } \\
\text { health and } 2=\text { worst } \\
\text { health }\end{array}$ & 30 \\
\hline $\begin{array}{l}\text { CIVIQ-20 [22, 24-26, } \\
29,30,37,39]\end{array}$ & CVI & $\begin{array}{l}\text { Physical, psycho- } \\
\text { logical and social } \\
\text { impairment, pain }\end{array}$ & 20 & $\begin{array}{l}\text { 5-point Likert scale, } \\
\text { ordinal }\end{array}$ & $\begin{array}{l}0-100, \text { with } \\
100=\text { worst QoL } \\
\text { and } 0=\text { best QoL }\end{array}$ & 28 \\
\hline $\begin{array}{l}\text { SF-36 }[20,21,28, \\
\quad 32-36,38]\end{array}$ & $\begin{array}{l}\text { Clinical practice and } \\
\text { research, health pol- } \\
\text { icy evaluations and } \\
\text { general popula- } \\
\text { tion surveys }\end{array}$ & $\begin{array}{l}\text { PF, PA, BP, GH, V, } \\
\text { SF, EWB, MH }\end{array}$ & 36 & NR & $\begin{array}{l}0-100 \text {, with } 0=\text { worst } \\
\text { QoL and } 100=\text { best } \\
\text { QoL }\end{array}$ & NR \\
\hline EQ-5D $[28,31,35]$ & NR & $\begin{array}{l}\text { Mobility, self-care, } \\
\text { usual activities, pain, } \\
\text { anxiety }\end{array}$ & 5 & $\begin{array}{l}\text { 3-point Likert scale, } \\
\text { ordinal }\end{array}$ & $\begin{array}{l}\text { Responses fitted into } \\
\text { an equation devel- } \\
\text { oped from a regres- } \\
\text { sion model which } \\
\text { produces a score } \\
\text { from }-0.59 \text { to } 1.00, \\
\text { with }-0.59=\text { worst } \\
\text { QoL, } 1.00=\text { best } \\
\text { QoL and } 0=\text { death }\end{array}$ & NR \\
\hline $\begin{array}{l}\text { PedsQL-NF1, adults } \\
\text { [23] }\end{array}$ & $\begin{array}{l}\text { Neurofibromatosis } \\
\text { type } 1\end{array}$ & $\begin{array}{l}\text { Physical, emotional, } \\
\text { social, cognitive } \\
\text { functioning, com- } \\
\text { munication, worry, } \\
\text { perceived physical } \\
\text { appearance, pain } \\
\text { and hurt, paresthe- } \\
\text { sia's, skin irritation, } \\
\text { sensation, movement } \\
\text { and balance, daily } \\
\text { activities, fatigue, } \\
\text { treatment anxiety, } \\
\text { sexual functioning }\end{array}$ & 74 & $\begin{array}{l}5 \text {-point likert scale, } \\
\text { ordinal }\end{array}$ & $\begin{array}{l}0-100, \text { with } 0=\text { worst } \\
\text { QoL and } 100=\text { best } \\
\text { QoL }\end{array}$ & NR \\
\hline $\begin{array}{l}\text { PedsQL-NF1, chil- } \\
\text { dren, adolescents } \\
\text { and young adults } \\
\text { [19] }\end{array}$ & $\begin{array}{l}\text { Neurofibromatosis } \\
\text { type } 1\end{array}$ & $\begin{array}{l}\text { Skin, pain, pain } \\
\text { impact, pain man- } \\
\text { agement, cognitive } \\
\text { functioning, speech, } \\
\text { fine motor, balance, } \\
\text { vision, perceived } \\
\text { physical appearance, } \\
\text { communication, } \\
\text { worry, treatment, } \\
\text { medicines, gastroin- } \\
\text { testinal symptoms }\end{array}$ & 115 & $\begin{array}{l}\text { 3-point likert scale, } \\
\text { ordinal }\end{array}$ & UD & UD \\
\hline
\end{tabular}

$L M F$ lymphatic malformation function instrument, CIVIQ-20 20-item chronic venous insufficiency quality of life questionnaire, $C V I$ chronic venous insufficiency, $Q o L$ quality of life, $S F-36$ Short-Form 36 health survey, $P F$ physical functioning, $P A$ physical aspects, $B P$ bodily pain, $G H$ general health, $V$ vitality, $S F$ social functioning, $E W B$ emotional well-being, $M H$ mental health, $N R$ not reported, $E Q-5 D$ EuroQuality of life 5-dimensions, $P e d s Q L-N F 1$ pediatric quality of life neurofibromatosis type 1, UD under development

inadequate structural validity, and for inadequate hypotheses testing. The evidence for test-retest reliability was unknown, as the analysis was only performed in seventeen patients and therefore the methodological quality of the study on reliability was poor [27]. Data on interpretability, responsiveness to changes over time or feasibility were lacking.

\section{0-item chronic venous insufficiency QoL questionnaire (CIVIQ-20)}

The CIVIQ-20 is a disease-specific HRQoL questionnaire for patients with chronic venous insufficiency (CVI), which was validated in patients with CVI [22, 24-26, 29, 30, 37] 


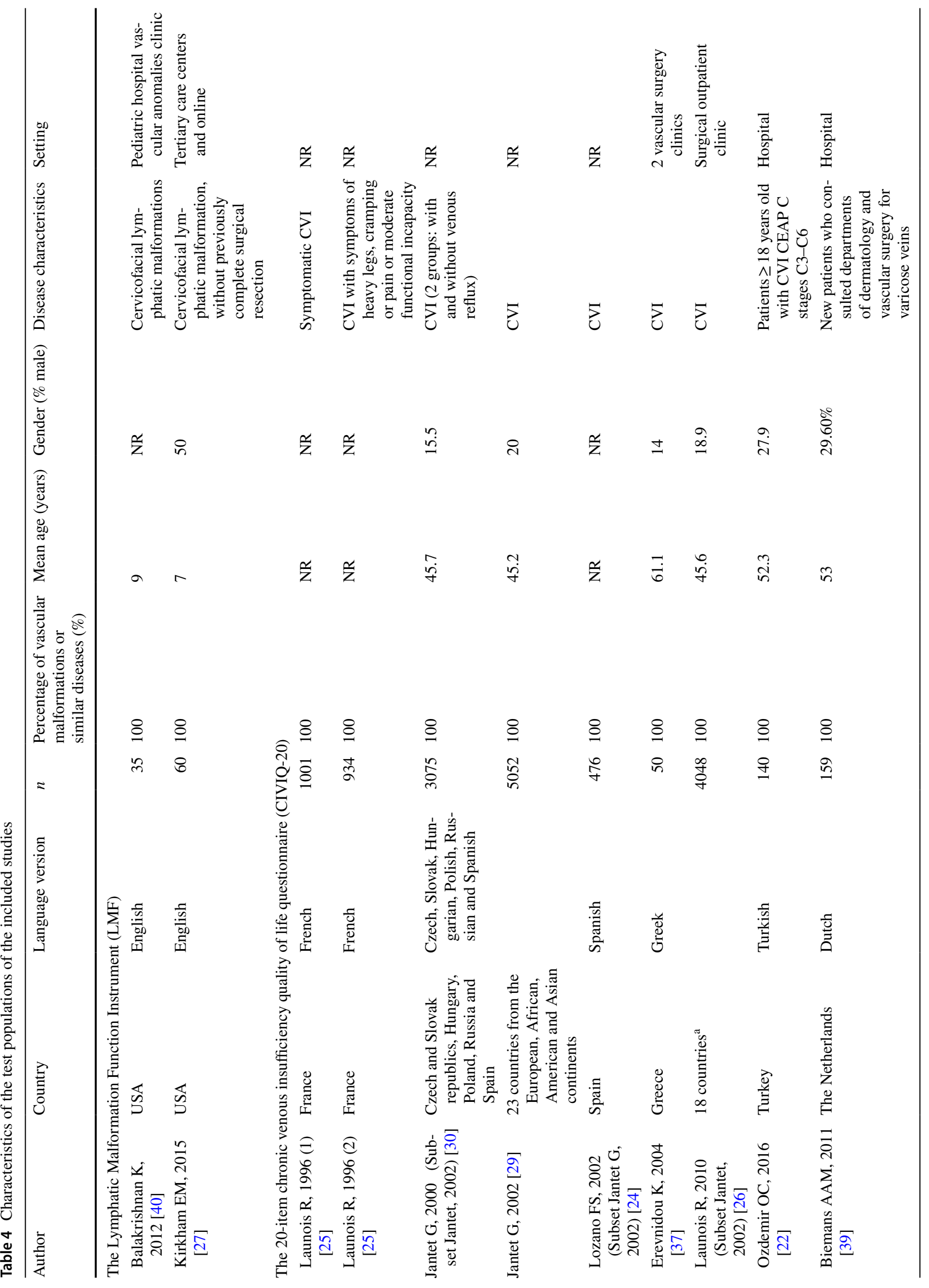




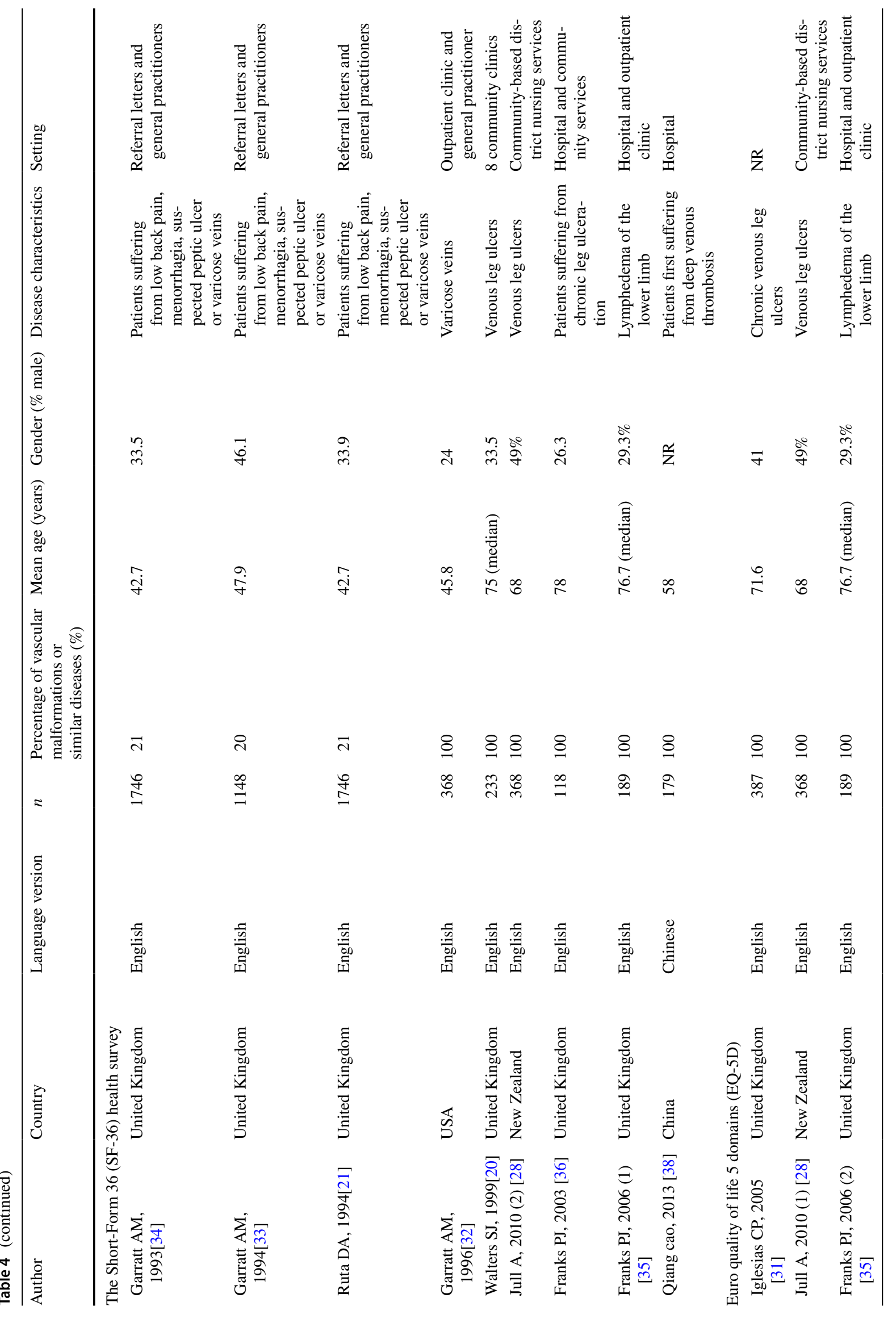


and in (isolated) varicose veins [39]. In CVI, moderate evidence was found for adequate internal consistency and reliability. The evidence for measurement error was rated 'indeterminate', as the minimal important change was not defined [22], although this is crucial for concluding if changes in score can be attributed to true changes in the construct. For chronic venous insufficiency, there was moderate evidence for adequate content validity and structural validity.

The evidence for adequate hypotheses-testing and responsiveness was rated as moderate (CVI) and limited (varicose veins), as the statistical methods used were suboptimal. Ceiling effects were found in three items, which may limit the instrument's ability to detect changes in health. The number of missing items was low (0-9.4\%).

\section{Short-Form-36 health survey version 2 (SF36v2)}

The SF-36 is a generic HRQoL questionnaire [20, 21, 28, $32-36,38]$. Evidence for adequate internal consistency varied from unknown (venous leg ulcers and lymphedema of the lower limb) [20, 35, 36], limited (varicose veins) [21, 34] to strong (deep venous thrombosis (DVT)) [38]. The evidence for adequate reliability was considered limited for varicose veins and moderate for DVT, as information on missing items was lacking. The evidence for structural validity could not be interpreted due to the lack of information about explained variance $[34,38]$. Finally, limited to moderate evidence for adequate responsiveness was found in lymphedema of the lower limb [35] and varicose veins [32, 33], respectively. There was conflicting evidence for venous leg ulcers [20, 28, 36], and in DVT the evidence was not interpretable since predefined hypotheses about the expected results were lacking [38]. In the studies describing interpretability [20,35], floor- and ceiling effects were found in three of eight dimensions. Feasibility aspects were not reported.

\section{EuroQoL-5-dimensions (EQ-5D)}

The EQ-5D is a generic HRQoL questionnaire, for which only 3 validation studies were performed in our target populations. In venous leg ulcers, limited evidence was available for inadequate hypotheses-testing for construct validity as no predefined hypotheses were stated for the expected correlations [31], whereas in lymphedema of the lower limb, limited evidence was found for adequate hypothesis-testing validity [35]. The evidence for adequate responsiveness was moderate and limited quality in venous leg ulcers and lymphedema of the lower limb, respectively, because the statistical methods applied were suboptimal or not appropriate [28, 31, 35]. No floor- and ceiling effects were found [35]. Feasibility aspects were not reported. 
Table 5 Methodological quality, quality assessment of measurement properties and best evidence synthesis per instrument and per disease

\begin{tabular}{|c|c|c|c|c|c|c|c|c|}
\hline Author, year & $\begin{array}{l}\text { Internal con- } \\
\text { sistency }\end{array}$ & Reliability & $\begin{array}{l}\text { Measurement } \\
\text { error }\end{array}$ & $\begin{array}{l}\text { Content valid- } \\
\text { ity }\end{array}$ & $\begin{array}{l}\text { Structural } \\
\text { validity }\end{array}$ & $\begin{array}{l}\text { Hypotheses } \\
\text { testing }\end{array}$ & $\begin{array}{l}\text { Cross- } \\
\text { cultural } \\
\text { validity }\end{array}$ & Responsiveness \\
\hline \multicolumn{9}{|c|}{ The lymphatic malformation function instrument (LMF) } \\
\hline \multicolumn{9}{|c|}{ Cervicofacial lymphatic malformations } \\
\hline $\begin{array}{l}\text { Bal- } \\
\text { akrishnan } \\
\text { K, } 2012 \\
{[40]}\end{array}$ & & & & Excellent $(+)$ & & & & \\
\hline $\begin{array}{l}\text { Kirkham } \\
\text { EM, } 2015 \\
{[27]}\end{array}$ & Fair $(+)$ & Poor $(+)$ & & & Fair $(-)$ & Fair $(-)$ & & \\
\hline $\begin{array}{l}\text { Best } \\
\text { evidence } \\
\text { synthesis }\end{array}$ & + & $?$ & NA & +++ & - & - & NA & NA \\
\hline \multicolumn{9}{|c|}{ The 20-item chronic venous insufficiency quality of life questionnaire (CIVIQ-20) } \\
\hline \multicolumn{9}{|c|}{ Chronic venous insufficiency } \\
\hline $\begin{array}{c}\text { Launois R, } \\
1996 \text { (1) } \\
{[25]}\end{array}$ & Fair $(+)$ & Fair (?) & & & Fair $(+)$ & Poor $(+)$ & & \\
\hline $\begin{array}{c}\text { Launois R, } \\
1996(2) \\
{[25]}\end{array}$ & Fair $(+)$ & & & Good $(+)$ & Fair $(+)$ & & & poor (?) \\
\hline $\begin{array}{l}\text { Jantet G, } \\
2000[30]\end{array}$ & Fair (+) & Fair (+) & & & Fair (?) & Poor (+) & Poor $(+)$ & Fair (?) \\
\hline $\begin{array}{l}\text { Jantet G, } \\
2002 \text { [29] }\end{array}$ & Poor $(+)$ & Fair $(+)$ & & & Poor (?) & Fair $(+)$ & & Fair $(+)$ \\
\hline $\begin{array}{r}\text { Lozano FS, } \\
2002[24]\end{array}$ & Fair (+) & Fair (+) & & & & & Poor (?) & Poor (?) \\
\hline $\begin{array}{c}\text { Erevnidou } \\
\text { K, } 2004 \\
{[37]}\end{array}$ & Fair (+) & Fair (?) & & & Poor (-) & Fair (+) & Poor (?) & \\
\hline $\begin{array}{l}\text { Launois R, } \\
2010[26]\end{array}$ & Good (+) & Good (+) & & & Good (?) & Good (+) & & Fair $(+)$ \\
\hline $\begin{array}{l}\text { Ozdemir } \\
\text { OC, } 2016 \\
{[22]}\end{array}$ & Poor (+) & Fair (+) & Fair (?) & & Fair (+) & Fair (+) & Fair (+) & \\
\hline $\begin{array}{l}\text { Best } \\
\text { evidence } \\
\text { synthesis }\end{array}$ & ++ & ++ & NI & ++ & ++ & ++ & + & ++ \\
\hline \multicolumn{9}{|l|}{ Varicose veins } \\
\hline $\begin{array}{l}\text { Biemans } \\
\text { AAM, } \\
2011 \text { [39] }\end{array}$ & Poor $(+)$ & Good (?) & & & Good (?) & Fair (+) & Good (-) & Fair (+) \\
\hline $\begin{array}{l}\text { Best } \\
\text { evidence } \\
\text { synthesis }\end{array}$ & $?$ & NI & NA & NA & NI & + & -- & + \\
\hline \multicolumn{9}{|c|}{ The Short-Form 36 (SF-36) health survey } \\
\hline \multicolumn{9}{|l|}{ Varicose veins } \\
\hline $\begin{array}{c}\text { Garratt AM, } \\
1993 \text { [34] }\end{array}$ & fair $(+)$ & & & & Fair (?) & Fair $(+)$ & & \\
\hline $\begin{array}{c}\text { Garratt AM, } \\
1994 \text { [33] }\end{array}$ & & & & & & & & Fair $(+)$ \\
\hline $\begin{array}{l}\text { Ruta DA, } \\
1994 \text { [21] }\end{array}$ & poor $(+)$ & fair (?) & & & & & & \\
\hline $\begin{array}{c}\text { Garratt AM, } \\
1996 \text { [32] }\end{array}$ & & fair $(+)$ & & & & Fair $(+)$ & & Fair $(+)$ \\
\hline
\end{tabular}


Table 5 (continued)

\begin{tabular}{|c|c|c|c|c|c|c|c|c|}
\hline Author, year & $\begin{array}{l}\text { Internal con- } \\
\text { sistency }\end{array}$ & Reliability & $\begin{array}{l}\text { Measurement } \\
\text { error }\end{array}$ & $\begin{array}{l}\text { Content valid- } \\
\text { ity }\end{array}$ & $\begin{array}{l}\text { Structural } \\
\text { validity }\end{array}$ & $\begin{array}{l}\text { Hypotheses } \\
\text { testing }\end{array}$ & $\begin{array}{l}\text { Cross- } \\
\text { cultural } \\
\text { validity }\end{array}$ & Responsiveness \\
\hline $\begin{array}{l}\text { Best } \\
\text { evidence } \\
\text { synthesis }\end{array}$ & + & + & NA & NA & NI & ++ & NA & ++ \\
\hline \multicolumn{9}{|c|}{ Venous leg ulcers } \\
\hline $\begin{array}{l}\text { Walters SJ, } \\
1999[20]\end{array}$ & poor $(+)$ & & & & & Good (+) & & Good (-) \\
\hline $\begin{array}{l}\text { Jull A, } 2010 \\
\text { (2) }[28]\end{array}$ & & & & & & & & Fair (+) \\
\hline $\begin{array}{l}\text { Franks PJ, } \\
2003[36]\end{array}$ & poor $(+)$ & & & & & & & Fair (?) \\
\hline $\begin{array}{l}\text { Best } \\
\text { evidence } \\
\text { synthesis }\end{array}$ & $?$ & NA & NA & NA & NA & ++ & NA & \pm \\
\hline \multicolumn{9}{|c|}{ Lymphedema of the lower limb } \\
\hline $\begin{array}{c}\text { Franks PJ, } \\
2006(1) \\
{[35]}\end{array}$ & Poor $(+)$ & & & & & Poor $(+)$ & & Fair $(+)$ \\
\hline $\begin{array}{l}\text { Best } \\
\text { evidence } \\
\text { synthesis }\end{array}$ & $?$ & & & & & $?$ & & + \\
\hline \multicolumn{9}{|c|}{ Deep venous thrombosis } \\
\hline $\begin{array}{l}\text { Qiang cao, } \\
2013 \text { [38] }\end{array}$ & Excellent $(+)$ & Good (+) & & & Good (?) & & & Poor (?) \\
\hline $\begin{array}{l}\text { Best } \\
\text { evidence } \\
\text { synthesis }\end{array}$ & +++ & ++ & NA & NA & NI & NA & NA & NI \\
\hline \multicolumn{9}{|c|}{ The euro quality of life -5 domain (EQ-5D) } \\
\hline \multicolumn{9}{|c|}{ Venous leg ulcers } \\
\hline $\begin{array}{r}\text { Iglesias CP, } \\
2005 \text { [31] }\end{array}$ & & & & & & Fair $(-)$ & & Fair $(+)$ \\
\hline $\begin{array}{l}\text { Jull A, } 2010 \\
\text { (1) }[28]\end{array}$ & & & & & & & & Fair $(+)$ \\
\hline $\begin{array}{l}\text { Best } \\
\text { evidence } \\
\text { synthesis }\end{array}$ & NA & NA & NA & NA & NA & - & NA & ++ \\
\hline \multicolumn{9}{|c|}{ Lymphedema of the lower limb } \\
\hline $\begin{array}{c}\text { Franks PJ, } \\
2006(2) \\
{[35]}\end{array}$ & & & & & & Fair $(+)$ & & Fair $(+)$ \\
\hline $\begin{array}{l}\text { Best } \\
\text { evidence } \\
\text { synthesis }\end{array}$ & NA & NA & NA & NA & NA & + & NA & + \\
\hline \multicolumn{9}{|c|}{ Pediatric quality of life inventory neurofibromatosis type 1 (PedsQL NF1, adults) } \\
\hline \multicolumn{9}{|c|}{ Neurofibromatosis type 1} \\
\hline $\begin{array}{c}\text { Nutakki K, } \\
2013 \text { (1) } \\
{[23]}\end{array}$ & Poor $(+)$ & & & Good $(+)$ & & & & \\
\hline $\begin{array}{c}\text { Nutakki K, } \\
2013 \text { (2) } \\
{[23]}\end{array}$ & Excellent (+) & & & & Excellent (?) & Fair $(+)$ & & \\
\hline $\begin{array}{l}\text { Best } \\
\text { evidence } \\
\text { synthesis }\end{array}$ & +++ & NA & NA & ++ & $?$ & + & NA & NA \\
\hline
\end{tabular}


Table 5 (continued)

\begin{tabular}{|c|c|c|c|c|c|c|c|c|}
\hline Author, year & $\begin{array}{l}\text { Internal con- } \\
\text { sistency }\end{array}$ & Reliability & $\begin{array}{l}\text { Measurement } \\
\text { error }\end{array}$ & $\begin{array}{l}\text { Content valid- } \\
\text { ity }\end{array}$ & $\begin{array}{l}\text { Structural } \\
\text { validity }\end{array}$ & $\begin{array}{l}\text { Hypotheses } \\
\text { testing }\end{array}$ & $\begin{array}{l}\text { Cross- } \\
\text { cultural } \\
\text { validity }\end{array}$ & Responsiveness \\
\hline \multicolumn{9}{|c|}{ Pediatric quality of life inventory neurofibromatosis type 1 (PedsQL NF1, children, adolescents and young adults) } \\
\hline \multicolumn{9}{|c|}{ Neurofibromatosis type 1} \\
\hline $\begin{array}{l}\text { Nutakki K, } \\
2017 \text { [19] }\end{array}$ & & & & Excellent $(+)$ & & & & \\
\hline $\begin{array}{l}\text { Best } \\
\text { evidence } \\
\text { synthesis }\end{array}$ & NA & NA & NA & +++ & NA & NA & NA & NA \\
\hline
\end{tabular}

For each measurement property, the methodological quality of the study is reported as 'poor', 'fair', 'good' or 'excellent'. The ratings of the study results of the analyses on this measurement property are depicted between brackets as '- '(negative result),'?'(indeterminate result), ' + ' (positive result). These two ratings were combined into the best evidence synthesis:,,++++++ positive rating indicating adequate measurement property; ? unknown rating indicating indeterminate measurement property;,,------ negative rating indicating inadequate measurement property; \pm conflicting findings, NI not interpretable (due to indeterminate result of analysis), NA not available. (analysis was not performed for this measurement property)

\section{Pediatric QoL inventory (PedsQL) -NF1, adults}

The PedsQL-NF1 is a disease-specific HRQoL questionnaire for patients with neurofibromatosis type 1 [23]. There was strong evidence for adequate internal consistency and moderate evidence for adequate content validity. Evidence for structural validity is unknown because no explained variance was presented in the included study [23]. Evidence for adequate hypotheses-testing validity was rated limited, as hypotheses were formulated in retrospect and not predefined. Feasibility, measured by the percentage of missing responses was $4.8 \%$ for all subscales [23]. Interpretability was not assessed.

\section{PedsQL-NF1, children, adolescents and young adults}

Finally, strong evidence for adequate content validity was found for the PedsQL-NF1 in children, adolescents and young adults [19, 23]. Information on other measurement properties is lacking.

\section{Discussion}

All eight outcome domains which were agreed on as the core outcome domains in the OVAMA e-Delphi study were assessed in previous prospective studies. However, the outcome domains are not measured consistently throughout studies, and the instruments used differ markedly as well.

The LMF is the only partially validated disease-specific instrument available that has been developed as a composite instrument to assess signs and subsequent impact on daily activity of living in pediatric patients with cervicofacial LMs. We found strong evidence for adequate content validity in patients with cervicofacial lymphatic malformations, which is promising. Yet, future studies in larger samples should further investigate the internal consistency, reliability, measurement error, structural validity, hypotheses-testing for construct validity, cross-cultural validity and responsiveness of this instrument. The LMF was only published recently and has not yet been used in prospective studies [27]. Responsiveness has not been investigated, which is a crucial aspect when evaluating treatment outcome. A disadvantage is the applicability to a subset of pediatric patients with cervicofacial LMs only. To broaden its applicability, further validation of this instrument in other types of cervicofacial vascular malformations would be necessary.

The other included validation studies only concerned HRQoL instruments that were either generic or developed for other conditions with clinical similarities to vascular malformations. Since these instruments were not validated for vascular malformations, we evaluated the measurement properties of these instruments in similar clinical populations. The generalizability of these results may be debatable, but it reflects the best available evidence for exploring which instruments show the greatest promise for use in vascular malformation research.

For assessing HRQoL, the SF-36 is a promising measure for adult patients as its measurement properties are wellinvestigated in diseases that are clinically similar to vascular malformations. Fewer validation studies in smaller patient populations with clinical similar diseases were available for the EQ-5D. The SF-10, FACT-G and FACIT were previously used for vascular malformations, but have not been validated for this condition or a similar disease. They may be equally applicable to vascular malformations in terms of item relevance, comprehensiveness and comprehensibility, but to date there is no evidence to support this. For children with vascular malformations, the PedsQL was the only HRQoL instrument used. This instrument was investigated 
in children and parents with neurofibromatosis and seems favorable with regard to the measurement properties analyzed so far [19, 23].

The CIVIQ-20 [41] has adequate measurement properties for patients with venous insufficiency and therefore it may be worthwhile to further investigate this instrument for vascular malformations of the lower extremities. This may also apply to other instruments for similar diseases that have not yet been used for vascular malformations, like VEINESQoL [42] or the Nottingham Health Profile [43] for varicose veins. However, the face and content validity of these instruments may be suboptimal for capturing the most important aspects for patients with vascular malformations, or may only be applicable to a specific type or location of the vascular malformation.

Interestingly, all included validation studies used classical test theory (CTT) methods, as opposed to item response theory (IRT). In CTT, measurement properties are assessed on instrument-level, depending on the items and study sample used, whereas IRT has an item-level focus [44]. The individual validation of items in IRB-based instruments facilitates computer-adaptive testing, in which the items used in the questioning process adapt to the respondent's previous answers [45], and linkage with existing item banks such as the 'Patient-Reported Outcomes Measurement Information System' [PROMIS] [46].

There were no validation studies available for instruments measuring the patient- or physician-reported core domains for vascular malformations pain, overall severity of symptoms, patient satisfaction with treatment and outcome nor for the recommended domains appearance and recurrence. Radiologic assessment of the vascular malformation fell outside the scope of this study as we focused on patient/physician-subjective instruments. Further research is required to determine which radiologic imaging modalities are suitable for measuring treatment outcome.

It seems necessary to develop a new disease-specific instrument for vascular malformations, or a disease-specific attribution module that can be used alongside a generic instrument, to adequately cover all previously established core domains. In general, disease-specific instruments are also more likely to pick up small differences in quality of life caused by disease burden than the broad generic instruments.

\section{Conclusion}

This study provides information on the available evidence for the quality of patient- and physician-reported outcome measurement instruments that have been developed or have previously been used for peripheral vascular malformations. The LMF is the only available disease-specific instrument for assessing signs and life impact in children with cervicofacial LMs. The identified generic HRQoL instruments, of which SF-36 (for adults) and PedsQL (for children) seem the most widely applicable, most investigated and promising in terms of measurement properties, may be used but it remains unclear if these instruments are responsive to treatment-induced changes in health in patients with vascular malformations. Further research into measurement properties may therefore be necessary to assess if the instruments that were identified in this systematic review are suitable for inclusion in the COS. It is likely that new diseasespecific instruments need to be developed to adequately cover all core domains for vascular malformations.

The results of this review will be used as input for the future consensus meeting with all stakeholders aiming to reach consensus on the core outcome measurement instruments.

Acknowledgements F. Blei MD MBA ${ }^{5}$, C.J.M. van der Vleuten MD $\mathrm{PhD}^{6}$, I. J. Frieden $\mathrm{MD}^{7}$, G.T. Richter $\mathrm{MD}^{8}$, S.T. Tan MBBS PhD FRACS ${ }^{9}$, T. Muir MBChB, Mmed (Plast Surg) ${ }^{10}$, A.J. Penington MD ${ }^{11}$, L.M. Boon MD PhD ${ }^{12}$; ${ }^{5}$ Department of Pediatrics, Lenox Hill Hospital of Northwell Health, New York, USA; ${ }^{6}$ Department of Dermatology, Radboud University Medical Center, Nijmegen, The Netherlands; ${ }^{7}$ Department of Dermatology, University of California San Francisco, San Francisco, USA; ${ }^{8}$ Department of Otolaryngology, Arkansas Children's Hospital, Arkansas, USA; ${ }^{9}$ Wellington Regional Plastic, Maxillofacial and Burns Unit, Hutt Hospital, and Gillies McIndoe Research Institute, Wellington, New Zealand; ${ }^{10}$ Department of Plastic and Reconstructive Surgery, James Cook University Hospital, Middlesbrough, UK; ${ }^{11}$ Department of Paediatrics, University of Melbourne and Murdoch Children's Research Institute, Melbourne Australia; ${ }^{12}$ Center for Vascular Anomalies, Division of Plastic and Reconstructive Surgery, Cliniques Universitaires Saint-Luc, Brussel, Belgium

Funding The authors of this article did not receive any funding for this study.

\section{Compliance with ethical standards}

Conflict of interest All the authors declare that they have no conflict of interest.

Informed consent As this article concerns a systematic review of previously published studies, as opposed to an original study with human participants performed by the authors of this article, obtaining informed consent was not applicable.

Research involving human and animal participants This article does not contain any studies with human participants or animals performed by any of the authors.

Open Access This article is distributed under the terms of the Creative Commons Attribution 4.0 International License (http://creativeco mmons.org/licenses/by/4.0/), which permits unrestricted use, distribution, and reproduction in any medium, provided you give appropriate credit to the original author(s) and the source, provide a link to the Creative Commons license, and indicate if changes were made. 


\section{References}

1. Cahill, A. M., \& Nijs, E. L. (2011). Pediatric vascular malformations: Pathophysiology, diagnosis, and the role of interventional radiology. Cardiovascular and Interventional Radiology, 34(4), 691-704. https://doi.org/10.1007/s00270-011-0123-0.

2. Garzon, M. C., Huang, J. T., Enjolras, O., \& Frieden, I. J. (2007). Vascular malformations: Part I. Journal of the American Academy of Dermatology, 56(3), 353-370. https://doi.org/10.1016/j. jaad.2006.05.069. (quiz 371-354).

3. Sierre, S., Teplisky, D., \& Lipsich, J. (2016). Vascular malformations: An update on imaging and management. Archivos Argentinos de Pediatria, 114(2), 167-176. https://doi. org/10.5546/aap.2016.eng.167.

4. Wassef, M., Blei, F., Adams, D., Alomari, A., Baselga, E., Berenstein, A., et al. (2015). Vascular anomalies classification: Recommendations from the International Society for the Study of Vascular Anomalies. Pediatrics, 136(1), e203-214. https:// doi.org/10.1542/peds.2014-3673.

5. Clarke, M. (2007). Standardising outcomes for clinical trials and systematic reviews. Trials, 8, 39. https://doi. org/10.1186/1745-6215-8-39.

6. Horbach, S. E. R., van der Horst, C., Blei, F., van der Vleuten, C. J. M., Frieden, I. J., Richter, G. T., et al. (2017). Development of an international core outcome set for peripheral vascular malformations (OVAMA project). British Journal of Dermatology. https://doi.org/10.1111/bjd.16029.

7. Schmitt, J., Apfelbacher, C., Spuls, P. I., Thomas, K. S., Simpson, E. L., Furue, M., et al. (2015). The Harmonizing Outcome Measures for Eczema (HOME) roadmap: A methodological framework to develop core sets of outcome measurements in dermatology. Journal of Investigative Dermatology, 135(1), 24-30. https://doi.org/10.1038/jid.2014.320.

8. Moher, D., Shamseer, L., Clarke, M., Ghersi, D., Liberati, A., Petticrew, M., et al. (2015). Preferred reporting items for systematic review and meta-analysis protocols (PRISMAP) 2015 statement. Systematic Reviews, 4, 1. https://doi. org/10.1186/2046-4053-4-1.

9. Kirkham, J. J., Gorst, S., Altman, D. G., Blazeby, J. M., Clarke, M., Devane, D., et al. (2016). Core outcome set-standards for reporting: The COS-STAR statement. PLoS Medicine, 13(10), e1002148. https://doi.org/10.1371/journal.pmed.1002148.

10. Retreived from http://www.cosmin.nl/.

11. Enjolras, O., Wassef, M., \& Chapot, R. (2007). Color atlas of vascular tumors and vascular malformations. Cambridge: Cambridge University Press.

12. Terwee, C. B., Jansma, E. P., Riphagen, I. I., \& de Vet, H. C. (2009). Development of a methodological PubMed search filter for finding studies on measurement properties of measurement instruments. Quality of Life Research, 18(8), 1115-1123. https:// doi.org/10.1007/s11136-009-9528-5.

13. Mokkink, L. B., Terwee, C. B., Knol, D. L., Stratford, P. W., Alonso, J., Patrick, D. L., et al. (2010). The COSMIN checklist for evaluating the methodological quality of studies on measurement properties: A clarification of its content. BMC Medical Research Methodology, 10, 22. https://doi.org/10.1186/1471-2288-10-22.

14. Mokkink, L. B., Terwee, C. B., Patrick, D. L., Alonso, J., Stratford, P. W., Knol, D. L., et al. (2010). The COSMIN checklist for assessing the methodological quality of studies on measurement properties of health status measurement instruments: An international Delphi study. Quality of Life Research, 19(4), 539-549. https://doi.org/10.1007/s11136-010-9606-8.

15. Terwee, C. B., Mokkink, L. B., Knol, D. L., Ostelo, R. W., Bouter, L. M., \& de Vet, H. C. (2012). Rating the methodological quality in systematic reviews of studies on measurement properties: A scoring system for the COSMIN checklist. Quality of Life Research, 21(4), 651-657. https://doi.org/10.1007/s1113 6-011-9960-1.

16. Prinsen, C. A., Vohra, S., Rose, M. R., Boers, M., Tugwell, P., Clarke, M., et al. (2016). How to select outcome measurement instruments for outcomes included in a "Core Outcome Set"A practical guideline. Trials, 17(1), 449. https://doi.org/10.1186/ s13063-016-1555-2.

17. Terwee, C. B., Bot, S. D., de Boer, M. R., van der Windt, D. A., Knol, D. L., Dekker, J., et al. (2007). Quality criteria were proposed for measurement properties of health status questionnaires. Journal of Clinical Epidemiology, 60(1), 34-42. https:// doi.org/10.1016/j.jclinepi.2006.03.012.

18. Ono, Y., Osuga, K., Takura, T., Nakamura, M., Shibamoto, K., Yamamoto, A., et al. (2016). Cost-effectiveness analysis of percutaneous sclerotherapy for venous malformations. Journal of Vascular and Interventional Radiology, 27(6), 831-837. https:// doi.org/10.1016/j.jvir.2015.12.019.

19. Nutakki, K., Varni, J. W., Steinbrenner, S., Draucker, C. B., \& Swigonski, N. L. (2017). Development of the pediatric quality of life inventory neurofibromatosis type 1 module items for children, adolescents and young adults: Qualitative methods. Journal of Neuro-oncology, 132(1), 135-143. https://doi.org/10.1007/s1106 0-016-2351-2.

20. Walters, S. J., Morrell, C. J., \& Dixon, S. (1999). Measuring health-related quality of life in patients with venous leg ulcers. Quality of Life Research, 8(4), 327-336.

21. Ruta, D. A., Abdalla, M. I., Garratt, A. M., Coutts, A., \& Russell, I. T. (1994). SF 36 health survey questionnaire: I. Reliability in two patient based studies. Quality of Health Care, 3(4), 180-185.

22. Ozdemir, O. C., Tonga, E., Tekindal, A., \& Bakar, Y. (2016). Cross-cultural adaptation, reliability and validity of the Turkish version of the chronic venous disease quality of life questionnaire (CIVIQ-20). Springerplus, 5, 381. https://doi.org/10.1186/s4006 4-016-2039-2.

23. Nutakki, K., Hingtgen, C. M., Monahan, P., Varni, J. W., \& Swigonski, N. L. (2013). Development of the adult PedsQL neurofibromatosis type 1 module: Initial feasibility, reliability and validity. Health Quality of Life Outcomes, 11, 21. https://doi. org/10.1186/1477-7525-11-21.

24. Lozano, F. S., \& Launois, R. (2002). Quality of life (Spain and France): Validation of the chronic venous insufficiency questionnaire (CIVIQ). Methods and Findings in Experimental and Clinical Pharmacology, 24(7), 425-429.

25. Launois, R., Reboul-Marty, J., \& Henry, B. (1996). Construction and validation of a quality of life questionnaire in chronic lower limb venous insufficiency (CIVIQ). Quality of Life Research, 5(6), $539-554$.

26. Launois, R., Mansilha, A., \& Jantet, G. (2010). International psychometric validation of the chronic venous disease quality of life questionnaire (CIVIQ-20). European Journal of Vascular and Endovascular Surgery, 40(6), 783-789. https://doi.org/10.1016/j. ejvs.2010.03.034.

27. Kirkham, E. M., Edwards, T. C., Weaver, E. M., Balakrishnan, K., \& Perkins, J. A. (2015). The lymphatic malformation function (LMF) Instrument. Otolaryngology-Head and Neck Surgery, 153(4), 656-662. https://doi.org/10.1177/0194599815594776.

28. Jull, A., Parag, V., Walker, N., \& Rodgers, A. (2010). Responsiveness of generic and disease-specific health-related quality of life instruments to venous ulcer healing. Wound Repair and Regeneration, 18(1), 26-30. https://doi.org/10.1111/j.1524475X.2009.00556.x.

29. Jantet, G. (2002). Chronic venous insufficiency: Worldwide results of the RELIEF study. Reflux assessment and quality of life improvement with micronized Flavonoids. Angiology, 53(3), 245-256. https://doi.org/10.1177/000331970205300301. 
30. Jantet, G. (2000). RELIEF study: First consolidated European data. Reflux assessment and quality of life improvement with micronized Flavonoids. Angiology, 51(1), 31-37. https://doi. org/10.1177/000331970005100107.

31. Iglesias, C. P., Birks, Y., Nelson, E. A., Scanlon, E., \& Cullum, N. A. (2005). Quality of life of people with venous leg ulcers: A comparison of the discriminative and responsive characteristics of two generic and a disease specific instruments. Quality of Life Research, 14(7), 1705-1718.

32. Garratt, A. M., Ruta, D. A., Abdalla, M. I., \& Russell, I. T. (1996). Responsiveness of the SF-36 and a condition-specific measure of health for patients with varicose veins. Quality of Life Research, 5(2), 223-234.

33. Garratt, A. M., Ruta, D. A., Abdalla, M. I., \& Russell, I. T. (1994). SF 36 health survey questionnaire: II. Responsiveness to changes in health status in four common clinical conditions. Quality of Health Care, 3(4), 186-192.

34. Garratt, A. M., Ruta, D. A., Abdalla, M. I., Buckingham, J. K., \& Russell, I. T. (1993). The SF36 health survey questionnaire: An outcome measure suitable for routine use within the NHS? BMJ, 306(6890), 1440-1444.

35. Franks, P. J., Moffatt, C. J., Doherty, D. C., Williams, A. F., Jeffs, E., \& Mortimer, P. S. (2006). Assessment of health-related quality of life in patients with lymphedema of the lower limb. Wound Repair and Regeneration, 14(2), 110-118. https://doi.org/10.111 1/j.1743-6109.2006.00099.x.

36. Franks, P. J., McCullagh, L., \& Moffatt, C. J. (2003). Assessing quality of life in patients with chronic leg ulceration using the Medical Outcomes Short Form-36 questionnaire. Ostomy/Wound Management, 49(2), 26-37.

37. Erevnidou, K., Launois, R., Katsamouris, A., \& Lionis, C. (2004). Translation and validation of a quality of life questionnaire for chronic lower limb venous insufficiency into greek. International Angiology, 23(4), 394-399.

38. Cao, Q., Tian, Y., \& Sai, L. M. (2013). Roles of short form-36 health survey scale in the evaluation of quality of life in patients with deep vein thrombosis. Zhonghua Yi Xue Za Zhi, 93(21), 1615-1618.

39. Biemans, A. A., van der Velden, S. K., Bruijninckx, C. M., Buth, J., \& Nijsten, T. (2011). Validation of the chronic venous insufficiency quality of life questionnaire in Dutch patients treated for varicose veins. European Journal of Vascular and Endovascular Surgery, 42(2), 246-253. https://doi.org/10.1016/j. ejvs.2011.04.007.

40. Balakrishnan, K., Edwards, T. C., \& Perkins, J. A. (2012). Functional and symptom impacts of pediatric head and neck lymphatic malformations: Developing a patient-derived instrument. Otolaryngology-Head and Neck Surgery, 147(5), 925-931. https://doi. org/10.1177/0194599812450838.

41. Weitz-Tuoretmaa, A., Rautio, R., Valkila, J., Keski-Santti, H., Keski-Nisula, L., \& Laranne, J. (2014). Efficacy of OK-432 sclerotherapy in treatment of lymphatic malformations: Long-term follow-up results. European Archives of Oto-Rhino-Laryngology, 271(2), 385-390. https://doi.org/10.1007/s00405-013-2542-9.

42. Mean, M., Limacher, A., Kahn, S. R., \& Aujesky, D. (2014). The VEINES-QOL/Sym questionnaire is a reliable and valid diseasespecific quality of life measure for deep vein thrombosis in elderly patients. Quality of Life Research, 23(9), 2463-2471. https://doi. org/10.1007/s11136-014-0704-x.

43. Franks, P. J., \& Moffatt, C. J. (2001). Health related quality of life in patients with venous ulceration: use of the Nottingham health profile. Quality of Life Research, 10(8), 693-700.

44. Embretson, S., \& Yang, X. (2006). Item response theory. New Jersey: Lawrence Erlbaum Associates Publishers.
45. Robertson, D., \& Williams, G. H. (2009). Clinical and translational science: Principles of human research. New York: Academic Press.

46. Garcia, S. F., Cella, D., Clauser, S. B., Flynn, K. E., Lad, T., Lai, J.-S., et al. (2007). Standardizing patient-reported outcomes assessment in cancer clinical trials: A patient-reported outcomes measurement information system initiative. Journal of Clinical Oncology, 25(32), 5106-5112.

47. Terwee, C. B., \& Mokkink, L. (2011). Protocol for systematic reviews of measurement properties. Quality of Life Research, 21(4), 651-657.

48. Saddal, N. S., Sharif, A., Ahmad, S., Mirza, F., Akhtar, N., Anwar Ul, H., et al. (2007). Intralesional bleomycin injection a primary therapy for peripheral lymphangiomas. Pakistan Journal of Medical Sciences, 23(2), 220-222.

49. Choi, Y. H., Han, M. H., Kwon, O. K., Cha, S. H., \& Chang, K. H. (2002). Craniofacial cavernous venous malformations: Percutaneous sclerotherapy with use of ethanolamine oleate. Journal of Vascular and Interventional Radiology, 13(5), 475-482.

50. Li, J., Chen, J., Zheng, G., Liao, G., Fu, Z., Li, J., et al. (2010). Digital subtraction angiography-guided percutaneous sclerotherapy of venous malformations with pingyangmycin and/or absolute ethanol in the maxillofacial region. Journal of Oral and Maxillofacial Surgery, 68(9), 2258-2266. https://doi.org/10.1016/j. joms.2009.06.024.

51. Jerjes, W., Upile, T., Alexander Mosse, C., Hamdoon, Z., Morcos, M., Morley, S., et al. (2011). Prospective evaluation of 110 patients following ultrasound-guided photodynamic therapy for deep seated pathologies. Photodiagnosis and Photodynamic Therapy, 8(4), 297-306. https://doi.org/10.1016/j.pdpdt.2011.08.002.

52. Hassan, Y., Osman, A. K., \& Altyeb, A. (2013). Noninvasive management of hemangioma and vascular malformation using intralesional bleomycin injection. Annals of Plastic Surgery, 70(1), 70-73. https://doi.org/10.1097/SAP.0b013e31824e298d.

53. Ma, G., Lin, X. X., Li, W., Jin, Y. B., Wang, W., Hu, X. J., et al. (2007). Noninvasive laser therapy for skin superficial venous malformations with long pulsed Nd:YAG laser. Zhonghua Zheng Xing Wai Ke Za Zhi, 23(2), 83-86.

54. Sainsbury, D. C., Kessell, G., Fall, A. J., Hampton, F. J., Guhan, A., \& Muir, T. (2011). Intralesional bleomycin injection treatment for vascular birthmarks: A 5-year experience at a single United Kingdom unit. Plastic and Reconstructive Surgery, 127(5), 2031-2044. https://doi.org/10.1097/PRS.0b013e31820e923c.

55. Xu, D. P., Zhai, Q. K., Cheng, C., Gong, H., Wang, H. W., \& Wang, X. K. (2014). Appraisal of efficacy and safety of intralesional injection of high concentration of bleomycin A5 for treatment of huge macrocystic lymphatic malformations in cervical region. Journal of Craniofacial Surgery, 25(5), 1707-1709. https ://doi.org/10.1097/scs.0000000000000900.

56. Elsharawy, M. A., \& Moghazy, K. M. (2007). Surgical and endovascular management of arteriovenous malformation: Case series from a single center. Vascular, 15(3), 134-140.

57. Kella, N., Rathi, P. K., Sheikh, U., \& Qureshi, M. A. (2011). Our experience of bleomycin sclerotherapy for peripheral lymphangioma in children and review of the literature. Pakistan Journal of Medical Sciences, 27(1), 60-63.

58. Kumar, V., Kumar, P., Pandey, A., Gupta, D. K., Shukla, R. C., Sharma, S. P., et al. (2012). Intralesional bleomycin in lymphangioma: An effective and safe non-operative modality of treatment. Journal of Cutaneous and Aesthetic Surgery, 5(2), 133-136. https ://doi.org/10.4103/0974-2077.99456.

59. Muir, T., Kirsten, M., Fourie, P., Dippenaar, N., \& Ionescu, G. O. (2004). Intralesional bleomycin injection (IBI) treatment for haemangiomas and congenital vascular malformations. Pediatric Surgery International, 19(12), 766-773. https://doi.org/10.1007/ s00383-003-1058-6. 
60. Sofos, S., \& Liew, S. H. (2014). The use of long pulsed neodymium-doped yttrium aluminum garnet for the treatment of paediatric venous malformations. Annals of Dermatology, 26(4), 474-477. https://doi.org/10.5021/ad.2014.26.4.474.

61. Wu, H. W., Wang, X., Zheng, J. W., Zhao, H. G., Ge, J., Zhang, L., et al. (2016). Treatment of deep-seated facial microcystic lymphatic malformations with intralesional injection of pingyangmycin. Medicine (Baltimore), 95(37), e4790. https://doi.org/10.1097/ md.0000000000004790.

62. Zhong, P. Q., Zhi, F. X., Li, R., Xue, J. L., \& Shu, G. Y. (1998). Long-term results of intratumorous bleomycin-A5 injection for head and neck lymphangioma. Oral Surgery, Oral Medicine, Oral Pathology, Oral Radiology, and Endodontology, 86(2), 139-144.

63. Wohlgemuth, W. A., Muller-Wille, R., Teusch, V., Hammer, S., Wildgruber, M., \& Uller, W. (2016). Ethanolgel sclerotherapy of venous malformations improves health-related quality-of-life in adults and children-results of a prospective study. European Radiology. https://doi.org/10.1007/s00330-016-4603-0.

64. Adams, D. M., Trenor, C. C., 3rd, Hammill, A. M., Vinks, A. A., Patel, M. N., Chaudry, G., et al. (2016). Efficacy and safety of sirolimus in the treatment of complicated vascular anomalies. Pediatrics, 137(2), e20153257. https://doi.org/10.1542/ peds.2015-3257.

65. Dompmartin, A., Blaizot, X., Theron, J., Hammer, F., Chene, Y., Labbe, D., et al. (2011). Radio-opaque ethylcellulose-ethanol is a safe and efficient sclerosing agent for venous malformations. European Radiology, 21(12), 2647-2656. https://doi.org/10.1007/ s00330-011-2213-4.

66. Sannier, K., Dompmartin, A., Theron, J., Labbe, D., Barrellier, M. T., Leroyer, R., et al. (2004). A new sclerosing agent in the treatment of venous malformations. Study on 23 cases. Interventional Neuroradiology, 10(2), 113-127. https://doi.org/10.1177/15910 1990401000203
67. Schumacher, M., Dupuy, P., Bartoli, J. M., Ernemann, U., Herbreteau, D., Ghienne, C., et al. (2011). Treatment of venous malformations: First experience with a new sclerosing agent-a multicenter study. European Journal of Radiology, 80(3), e366-372. https://doi.org/10.1016/j.ejrad.2010.12.074.

68. Osuga, K., Hori, S., Kitayoshi, H., Khankan, A. A., Okada, A., Sugiura, T., et al. (2002). Embolization of high flow arteriovenous malformations: Experience with use of superabsorbent polymer microspheres. Journal of Vascular and Interventional Radiology, 13(11), 1125-1133.

69. Yamaki, T., Nozaki, M., Sakurai, H., Takeuchi, M., Soejima, K., \& Kono, T. (2008). Prospective randomized efficacy of ultrasoundguided foam sclerotherapy compared with ultrasound-guided liquid sclerotherapy in the treatment of symptomatic venous malformations. Journal of Vascular Surgery, 47(3), 578-584. https://doi. org/10.1016/j.jvs.2007.11.026.

70. Giguere, C. M., Bauman, N. M., Sato, Y., Burke, D. K., Greinwald, J. H., Pransky, S., et al. (2002). Treatment of lymphangiomas with OK-432 (Picibanil) sclerotherapy: A prospective multi-institutional trial. Archives of Otolaryngology-Head \& Neck Surgery, 128(10), 1137-1144.

71. Smith, M. C., Zimmerman, M. B., Burke, D. K., Bauman, N. M., Sato, Y., \& Smith, R. J. (2009). Efficacy and safety of OK-432 immunotherapy of lymphatic malformations. Laryngoscope, 119(1), 107-115. https://doi.org/10.1002/lary.20041.

Publisher's Note Springer Nature remains neutral with regard to jurisdictional claims in published maps and institutional affiliations.

\section{Affiliations}

\section{Sophie E. R. Horbach ${ }^{1} \cdot$ Amber P. M. Rongen ${ }^{1} \cdot$ Roy G. Elbers ${ }^{2} \cdot$ Chantal M. A. M. van der Horst $^{1} \cdot$ Cecilia A. C. Prinsen ${ }^{3}$. Phyllis I. Spuls ${ }^{4}$. Collaborators OVAMA consensus group}

1 Department of Plastic, Reconstructive and Hand Surgery, Academic Medical Center, University of Amsterdam, Amsterdam, The Netherlands

2 Department of Clinical Epidemiology, Biostatistics and Bioinformatics, Academic Medical Center, University of Amsterdam, Amsterdam, The Netherlands
3 Department of Epidemiology and Biostatistics, Amsterdam Public Health Research Institute, VU University Medical Centre, Amsterdam, The Netherlands

4 Department of Dermatology, Academic Medical Center, University of Amsterdam, Amsterdam, The Netherlands 